\title{
Updated patterns of failure after multimodality therapy for malignant pleural mesothelioma
}

\author{
Elizabeth H. Baldini, MD, MPH, ${ }^{\mathrm{a}}$ William G. Richards, PhD, ${ }^{\mathrm{b}}$ Ritu R. Gill, MD, MPH, \\ Brian M. Goodman, MD, ${ }^{d}$ Olivia K. Winfrey, MPH, ${ }^{\mathrm{e}}$ Hannah M. Eisen, MS, ${ }^{\mathrm{b}}$ Raymond H. Mak, MD, ${ }^{\mathrm{a}}$ \\ Aileen B. Chen, MD, MPP, ${ }^{\mathrm{a}}$ David E. Kozono, MD, PhD, ${ }^{\mathrm{a}}$ Raphael Bueno, MD, ${ }^{\mathrm{b}}$ and \\ David J. Sugarbaker, $\mathrm{MD}^{\mathrm{f}}$
}

\begin{abstract}
Objective: We have previously described patterns of failure after extrapleural pneumonectomy and multimodality therapy for malignant pleural mesothelioma and sought to update our results with a larger cohort of recent patients.

Methods: A total of 169 patients underwent extrapleural pneumonectomy without preoperative chemotherapy between 2001 and 2010. Data for treatment, recurrence, and survival were determined from medical records. A thoracic radiologist reviewed postoperative computed tomography or positron emission tomography computed tomography scans to determine sites of recurrence. Time to recurrence was estimated by the Kaplan-Meier method. Rates were compared using the Fisher exact test.
\end{abstract}

Results: The median age of patients was 62 years. Histology on final pathology was epithelial for 104 patients $(62 \%)$ and nonepithelial for 65 patients $(38 \%)$. A total of 132 patients $(78 \%$ ) received heated intraoperative chemotherapy; 77 patients $(45 \%)$ received adjuvant chemotherapy, and 71 patients $(42 \%)$ received adjuvant radiation therapy. Most chemotherapy regimens included platinum or pemetrexed. Median radiation therapy dose was $54 \mathrm{~Gy}$. Among 158 evaluable patients, a recurrence developed in $118(75 \%)$. Median follow-up was 83 months, median time to recurrence was 13.1 months, and median survival was 15 months. Sites of first recurrence were in the ipsilateral hemithorax or mediastinum for $54 \%$ of patients, in the abdomen for $39 \%$ of patients, in the contralateral hemithorax for $28 \%$ of patients, and in other distant sites for $5 \%$ of patients. Some patients had simultaneous recurrences in multiple sites.

Conclusions: The most common site of recurrence after extrapleural pneumonectomy and planned multimodality therapy remains the ipsilateral hemithorax (including mediastinum), and true distant failure (other than the abdomen or contralateral hemithorax) remains unusual. The distribution of recurrences is strikingly similar to our prior report. (J Thorac Cardiovasc Surg 2015;149:1374-81)

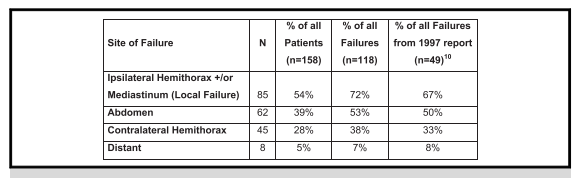

Sites of first failure for 158 patients in current cohort compared with previous cohort.

\section{Central Message}

Most common site of failure after EPP, heated intra-operative $\mathrm{CT}$, systemic CT, and RT remains the ipsilateral hemithorax.

\section{Perspective}

The most common site of recurrence after EPP and planned multimodality therapy remains the ipsilateral hemithorax and true distant failure (other than abdomen or contralateral hemithorax) remains unusual. The distribution of recurrences is strikingly similar to our previous report and highlights the need for aggressive adjunctive therapies that would be best tested on prospective multicenter trials.

See Editorial Commentary page 1382 .
Malignant pleural mesothelioma (MPM) is a rare but aggressive disease with poor prognosis. Median survival ranges from 7 months with supportive care to 12 months with chemotherapy, and perhaps longer with multimodality therapy including various combinations of surgery, radiation, and chemotherapy. ${ }^{1-6}$ There are numerous potential local

From the Department of Radiation Oncology, ${ }^{\text {a }}$ Brigham and Women's Hospital/ Dana-Farber Cancer Institute, Harvard Medical School, Boston, Mass; Division of Thoracic Surgery, ${ }^{b}$ and Department of Radiology, ${ }^{\mathrm{c}}$ Brigham and Women's Hospital, Harvard Medical School, Boston, Mass; Division of Thoracic Surgery, ${ }^{\mathrm{d}}$ Geisinger Health System, Wilkes-Barre, Pa; University of Kentucky College of Medicine, ${ }^{\mathrm{e}}$ Lexington, Ky; and Division of Thoracic Surgery, ${ }^{\mathrm{f}}$ Debakey Institute, Houston, Tex. and systemic treatment strategies for MPM, including extrapleural pneumonectomy (EPP), pleurectomy/ decortication, hemithoracic radiation therapy (RT), intracavitary and intravenous chemotherapy, photodynamic therapy, and novel targeted therapies. The optimal treatment approaches for various clinical scenarios of

Received for publication July 13, 2014; revisions received Sept 21, 2014; accepted for publication Oct 5, 2014; available ahead of print March 13, 2015

Address for reprints: Elizabeth H. Baldini, MD, MPH, Department of Radiation

Oncology, Brigham and Women's Hospital, L2, 75 Francis St, Boston, MA 02115 (E-mail: ebaldini@partners.org).

$0022-5223 / \$ 36.00$

Copyright (C) 2015 by The American Association for Thoracic Surgery

http://dx.doi.org/10.1016/j.jtcvs.2014.10.128 

Abbreviations and Acronyms
CHT $=$ contralateral hemithorax
$\mathrm{CI}=$ confidence interval
$\mathrm{CT}=$ computed tomography
$\mathrm{EPP}=$ extrapleural pneumonectomy
$\mathrm{EPT}=$ electron-photon technique
HIOC $=$ heated intraoperative chemotherapy
IHT $=$ ipsilateral hemithorax
IMRT $=$ intensity-modulated radiation therapy
MCR $=$ macroscopic complete resection
MPM = malignant pleural mesothelioma
PET = positron emission tomography
$\mathrm{RT}=$ radiation therapy

MPM have not been determined and are the subjects of active debate. ${ }^{7-9}$ However, it is clear that local control for disease confined to the ipsilateral hemithorax (IHT) is a major challenge, and for this reason, at Brigham and Women's Hospital and Dana-Farber Cancer Institute, we have pursued multimodality therapies including EPP for patients with localized node-negative MPM, nonsarcomatoid histology, and good performance status.

Understanding the specific patterns of failure after treatment strategies helps inform decisions regarding where to direct subsequent treatment efforts. In 1997, we reported patterns of failure for 49 patients after EPP, adjuvant chemotherapy (cyclophosphamide, doxorubicin, and cisplatin), and 2-dimensional RT. ${ }^{10}$ We found the most common site of failure was in the IHT, followed by failures in the abdomen, contralateral hemithorax (CHT), and, less frequently, in other distant sites. Since 1999, we have added heated intraoperative chemotherapy (HIOC) to our regimen in an attempt to augment local and abdominal control. ${ }^{11-13}$ In addition, the introduction of pemetrexed has established for the first time a standard-of-care regimen for intravenous chemotherapy. ${ }^{2}$ Another change is the routine availability of computed tomography (CT) scans for preoperative staging and post-treatment surveillance, which has improved both patient selection for surgery and detection of recurrent disease. Our practice also has incorporated specialist radiographic review. In the current analysis, we evaluate a separate and larger cohort of patients treated with intended multimodality therapy in the HIOC and pemetrexed era (between 2001 and 2010) to determine to what degree the patterns of failure have been affected by the evolution of MPM management since our prior analysis. Our group has recently described perioperative morbidity and mortality and overall survival for patients with epithelial MPM treated with EPP since $1988 .{ }^{14}$ Although some of the patients in the current cohort were included in the former report, the current report describes different outcomes, namely, patterns of failure for patients treated in the modern era, and is the first from Brigham and Women's Hospital and Dana-Farber Cancer Institute to describe recurrence patterns since 1997.

\section{MATERIALS AND METHODS}

A total of 447 patients who underwent EPP for MPM between 2001 and 2010 were identified from the Brigham and Women's Hospital International Mesothelioma Program Patient Data Registry. Unlike many institutions that use treatment strategies offering all patients neoadjuvant chemotherapy before resection, our strategy has been to treat eligible patients with initial surgery except in cases of mediastinoscopy-proven nodal involvement. For this reason, we have excluded patients treated with initial chemotherapy from the current analysis because they comprise a distinct cohort with poorer prognosis because of advanced stage at presentation. With institutional review board approval, records were reviewed to identify eligible patients who underwent macroscopic complete resection (MCR) by EPP at Brigham and Women's Hospital, who did not receive preoperative chemotherapy, and for whom a preoperative chest CT scan was available. A total of 169 patients met these criteria and constitute the study cohort.

After our prior experience with trimodality therapy, the intention-to-treat strategy during this study era was EPP with HIOC and subsequent evaluation for adjuvant chemotherapy and RT. Adjuvant chemotherapy or RT was recommended at the discretion of the treating oncologists and based on factors such as patient performance status, relative concerns of potential cumulative toxicity, and patient choice. EPP involved MCR achieved by en bloc resection of the lung, parietal pleura, ipsilateral pericardium, and ipsilateral diaphragm. Limited resection was also performed for previous biopsy, chest tube sites, or areas of localized gross tumor. Further details of the EPP technique have been reported. ${ }^{15}$ HIOC entailed intraoperative instillation into the ipsilateral chest and abdomen of heated cisplatin with or without gemcitabine. HIOC was delivered on 1 of 2 treatment protocols active during the study time period or off protocol using protocol-established safe and effective dose and administration parameters. These parameters included MCR followed by cisplatin 175 to $225 \mathrm{mg} / \mathrm{m}^{2}$ for a 1 -hour lavage at $42^{\circ} \mathrm{C}$ with sodium thiosulfate rescue and with or without amifostine protection. $^{12,13}$ Reasons that some patients did not receive HIOC included ineligibility related to renal function, complete blood count or other factors, lack of an available spot on protocol because of a waiting period, and patient refusal. Adjuvant therapy was initiated 6 to 12 weeks postoperatively. Systemic chemotherapy agents typically included cisplatin, pemetrexed, or both given every 3 weeks. Adjuvant RT to the hemithorax was delivered using previously described techniques, including a matched electron-photon technique (EPT) during the earlier years of the study period and intensity-modulated RT (IMRT) during the later years. ${ }^{16,17}$ Some patients treated at outside institutions received other 3-dimensional conformal RT techniques. Follow-up was typically a physical examination and chest CT or positron emission tomography (PET)/CT scan every 3 to 4 months for the first 3 years and every 6 months, thereafter.

Central review of all preoperative CT scans and all available postoperative studies (mostly CT scans and PET/CT scans) was performed by a dedicated thoracic radiologist (R.R.G.) to determine sites of recurrence. All sites of first recurrence were recorded and categorized as involving the IHT or mediastinum (local), CHT, abdomen, or other distant sites. Recurrence was scored as present if detected on imaging or physical examination. Biopsy or cytology confirmation was performed for many cases, but was not required in an effort to avoid under-reporting recurrences.

Recurrence-free interval was calculated as the time from surgery to first radiographic recurrence or biopsy determination of recurrence, with censoring on the date of the most recent study if recurrence was not demonstrated or the patient was subsequently lost to follow-up, or on the date of death if death from another cause (other than mesothelioma) was documented. Local recurrence-free survival was calculated as the time from surgery to the time of local recurrence, with censoring at the time 
of other sites of recurrence, death, or on the most recent contact date. Disease-free survival was calculated as the time from surgery to the time of any recurrence or death, with censoring for living patients on the most recent evaluation date. Overall survival was calculated as the time from surgery to the time of death from any cause, with censoring for living patients and those lost to follow-up on the most recent contact date. Kaplan-Meier survival function estimates were calculated and compared using the log-rank statistic. Proportions were compared using the Fisher exact test. Cox regression was performed to assess time to local recurrence and local recurrence-free survival, controlling for nonepithelial histology and use of RT, HIOC, and adjuvant chemotherapy.

\section{RESULTS \\ Preoperative Staging}

All patients had preoperative chest CT scans as a criterion for inclusion in this study cohort. Magnetic resonance imaging was consistently used to evaluate chest wall and diaphragmatic invasion except when precluded by metallic implants or claustrophobia (156/169 patients; $92 \%$ ). Mediastinoscopy was routinely performed after pemetrexed-cisplatin became established as the first standard of care chemotherapy in 2004 (133/136 cohort patients; $98 \%$ ), but had been infrequently used before that time $(2 / 33 ; 6 \%)$. Coincident with the addition of mediastinoscopy to the standard preoperative evaluation, PET/CT imaging became more readily available and was also incorporated into the standard workup to rule out extrathoracic metastasis $(123 / 136 ; 90 \%$ after 2004 vs $9 / 33 ; 27 \%$ before 2004).

\section{Clinical and Treatment Characteristics}

Patient, tumor, and treatment characteristics are shown in Table 1. Median age was 62 years (range, 37-79 years), and there were 136 men $(80 \%)$ and 33 women $(20 \%)$. Histologic subtype on initial pathology was epithelioid or biphasic for all patients, but on final pathology (at the time of EPP) was epithelioid for 104 patients $(62 \%)$, biphasic for 60 patients $(36 \%)$, sarcomatoid for 4 patients $(2 \%)$, and desmoplastic for 1 patient $(1 \%)$. All patients underwent EPP, and 132 patients $(78 \%)$ received HIOC. A total of 77 patients $(46 \%)$ received adjuvant chemotherapy; $85 \%$ of regimens included cisplatin, $79 \%$ contained pemetrexed, and $46 \%$ contained both. Seventy-one patients (42\%) received RT with a median dose of 54 Gy (range, 30-60.4 Gy). The RT technique was EPT for 31 patients (44\%), IMRT for 21 patients $(30 \%)$, and other 3-dimensional conformal techniques for 8 patients $(11 \%)$.

\section{Outcomes and Patterns of Failure}

A total of 156 patients are deceased, and 13 patients are alive with a median follow-up of 83 months (range, 58-107 months). Median overall survival among all 169 patients was 15.0 months. Detailed recurrence evaluation was not available for 11 patients ( 2 patients had recurrence, but the specific location of recurrence was not known, and 9
TABLE 1. Patient, tumor, and treatment characteristics for 169 patients with malignant pleural mesothelioma who underwent extrapleural pneumonectomy and adjuvant therapy

\begin{tabular}{|c|c|}
\hline Age & Median, 62 y (range, $37-79$ y) \\
\hline \multicolumn{2}{|l|}{ Gender } \\
\hline Men & $136(80 \%)$ \\
\hline Women & $33(20 \%)$ \\
\hline \multicolumn{2}{|l|}{ Side of disease } \\
\hline Right & $84(50 \%)$ \\
\hline Left & $85(50 \%)$ \\
\hline \multicolumn{2}{|c|}{ Pathologic AJCC stage } \\
\hline I & $4(2 \%)$ \\
\hline II & $25(15 \%)$ \\
\hline III & $89(53 \%)$ \\
\hline IV & $51(30 \%)$ \\
\hline \multicolumn{2}{|l|}{ pT classification } \\
\hline T0 & $1(1 \%)$ \\
\hline $\mathrm{T} 1$ & $4(2 \%)$ \\
\hline $\mathrm{T} 2$ & $39(23 \%)$ \\
\hline $\mathrm{T} 3$ & $74(44 \%)$ \\
\hline $\mathrm{T} 4$ & $51(30 \%)$ \\
\hline \multicolumn{2}{|l|}{ pN classification } \\
\hline N0 & $86(51 \%)$ \\
\hline N1 & $31(18 \%)$ \\
\hline $\mathrm{N} 2$ & $52(31 \%)$ \\
\hline \multicolumn{2}{|c|}{ Histology by final pathology } \\
\hline Epithelial & $104(62 \%)$ \\
\hline Biphasic & $60(36 \%)$ \\
\hline Sarcomatoid & $4(2 \%)$ \\
\hline Desmoplastic & $1(1 \%)$ \\
\hline \multicolumn{2}{|l|}{ Surgery } \\
\hline EPP & $169(100 \%)$ \\
\hline \multicolumn{2}{|l|}{ Adjuvant therapy } \\
\hline HIOC & $47(28 \%)$ \\
\hline HIOC, CTX & $26(15 \%)$ \\
\hline HIOC, RT & $21(12 \%)$ \\
\hline HIOC, CTX, RT & $38(22 \%)$ \\
\hline CTX, RT & $6(4 \%)$ \\
\hline CTX & $7(4 \%)$ \\
\hline RT & $6(4 \%)$ \\
\hline None & $18(11 \%)$ \\
\hline
\end{tabular}

$\overline{A J C C}$, American Joint Committee on Cancer; EPP, extrapleural pneumonectomy; $H I O C$, heated intraoperative chemotherapy; $C T X$, chemotherapy; $R T$, radiotherapy.

patients died perioperatively before evaluation for recurrence). Among 158 patients who were evaluable for recurrence, 118 had documented recurrence $(75 \%)$, with a median time to any recurrence of 13.1 months and median time to local recurrence of 18 months from the date of surgery. Actuarial 3-year local recurrence-free survival was $17 \%$ (95\% confidence interval [CI], 11-23), diseasefree survival was $16 \%(95 \% \mathrm{CI}, 10-21)$, and overall survival was $23 \%$ (95\% CI, 17-30).

Sites of first failure are depicted in Table 2. Some patients had recurrence in multiple locations. A total of 85 patients $(54 \%)$ had initial failure in sites that included the IHT or mediastinum (local failure), 62 patients (39\%) had initial 
TABLE 2. Sites of first failure among 158 patients evaluable for recurrence: Some patients had failures in more than 1 site

\begin{tabular}{lcccc}
\hline \multicolumn{1}{c}{ Site of failure } & N & $\begin{array}{c}\% \text { of all patients } \\
(\mathbf{n = 1 5 8})\end{array}$ & $\begin{array}{c}\% \text { of all failures } \\
(\mathbf{n}=\mathbf{1 1 8})\end{array}$ & $\begin{array}{c}\% \text { of all failures from 1997 report } \\
(\mathbf{n}=\mathbf{4 9})\end{array}$ \\
\hline IHT +/or mediastinum (local failure) & 85 & 54 & 72 & 67 \\
Abdomen & 62 & 39 & 53 & 50 \\
CHT & 45 & 28 & 38 & 33 \\
Distant & 8 & 5 & 7 & 8 \\
\hline
\end{tabular}

IHT, Ipsilateral hemithorax; $C H T$, contralateral hemithorax.

failure in the abdomen, 45 patients $(28 \%)$ had initial failure in the CHT, and 8 patients (5\%) had initial failure in other distant sites. The proportions of patients experiencing initial recurrence in the mediastinum, abdomen, or distant sites increased with pathologic stage, whereas the proportions recurring in the ipsilateral or CHT were consistent across stages (Figure 1). Detailed local recurrence rates were $47 \%$ in the IHT, $27 \%$ in the mediastinum, and $15 \%$ in the IHT or mediastinum only (without other sites of recurrence). Among all failures, $72 \%$ were local in the IHT or mediastinum, $53 \%$ were in the abdomen, $38 \%$ were in the CHT, and 7\% were in other distant sites.

Specific sites of first recurrence are shown in Table 3. The most common sites of recurrence within each site were as follows. For the IHT, $71 \%$ of recurrences were as a chest wall mass and $29 \%$ were neopleural thickening or mass. For the mediastinum, $70 \%$ were lymphadenopathy and $28 \%$ were as a soft tissue mass (that did not clearly represent lymphadenopathy.) For the abdomen, $31 \%$ were ascites, $27 \%$ were retroperitoneal adenopathy, and $24 \%$ were an abdominal mass. For the CHT, $49 \%$ were lung nodules and $49 \%$ were pleural effusions. The other distant sites included bone for $88 \%$, soft tissue for $13 \%$, and brain for $13 \%$.

Patients who received RT experienced a lower rate of local failure (in the IHT or mediastinum). Crude local recurrence was 39\% (27/70) among patients who received RT compared with $66 \%(58 / 88)$ among those who did not receive $\mathrm{RT}(P=.01)$, and actuarial 3-year local recurrence rate was $47 \%(95 \% \mathrm{CI}, 31-63)$ among patients who received RT compared with $82 \%$ (72\%-92\%) among those who did not $(P<.0001)$. Adjuvant RT was also associated with significantly longer time to local recurrence and local recurrence-free survival when correcting for histology, HIOC, and adjuvant chemotherapy (Table 4).

\section{DISCUSSION}

Our report from 1997 described patterns of failure after EPP and adjuvant cyclophosphamide, doxorubicin, and cisplatin chemotherapy and 2-dimensional conformal RT. ${ }^{10}$ Although there remains no clear current standard of care, our more recent treatment approach has consisted of a revised intention-to-treat strategy consisting of EPP, HIOC, and adjuvant pemetrexed-based chemotherapy and 3-dimensional RT or IMRT. In this updated series, we sought to determine whether our revised approach was associated with a change in the patterns of failure. We have demonstrated that for the current series of 169 patients treated with EPP between 2001 and 2010, recurrent disease developed in $75 \%$ of patients. The pattern of recurrence was local for $54 \%$ of patients, in the abdomen for $39 \%$ of patients, in the CHT for $28 \%$ of patients, and in other

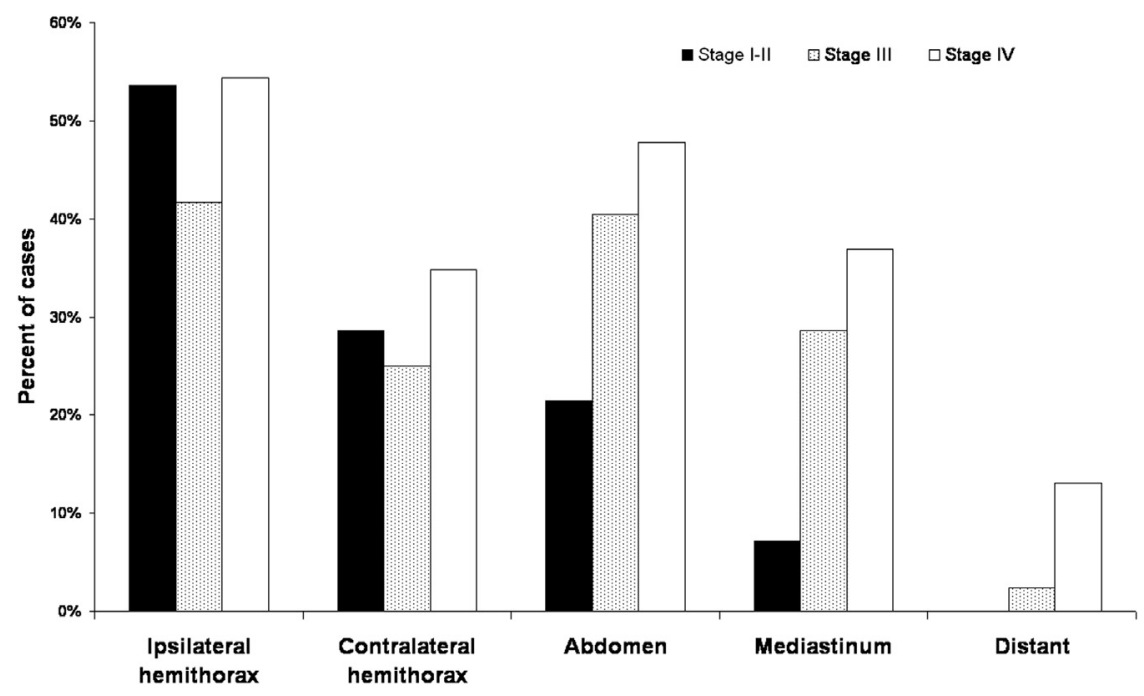

FIGURE 1. Percentage of patients with first site of recurrence in IHT, CHT, abdomen, and mediastinum according to the American Joint Committee on Cancer stage. 
TABLE 3. Detailed sites of first failures among 158 evaluable patients: Some patients had failures in more than 1 site

\begin{tabular}{|c|c|c|}
\hline Site & $\mathbf{N}$ & ( $\%$ of failures in that site) \\
\hline IHT (all failures) & 75 & \\
\hline Chest wall mass & 53 & 71 \\
\hline Neo-pleural thickening or mass & 22 & 29 \\
\hline Lymphadenopathy & 7 & 9 \\
\hline Pericardial thickening & 4 & 5 \\
\hline Mediastinum (all failures) & 43 & \\
\hline Lymphadenopathy & 30 & 70 \\
\hline Soft tissue mass & 12 & 28 \\
\hline Pericardial & 5 & 12 \\
\hline Abdomen (all failures) & 62 & \\
\hline Ascites & 19 & 31 \\
\hline Retroperitoneal adenopathy & 17 & 27 \\
\hline Abdominal mass NOS & 15 & 24 \\
\hline Liver & 12 & 19 \\
\hline Peritoneal mass & 11 & 18 \\
\hline Abdominal wall mass & 11 & 18 \\
\hline Mesenteric mass & 7 & 11 \\
\hline Omental mass & 7 & 11 \\
\hline CHT (all failures) & 45 & \\
\hline Lung nodules & 22 & 49 \\
\hline Pleural effusion & 22 & 49 \\
\hline Pleural thickening or mass & 6 & 13 \\
\hline Lymphadenopathy & 3 & 7 \\
\hline Other distant sites (all failures) & 8 & \\
\hline Bone & 7 & 88 \\
\hline Soft tissue & 1 & 13 \\
\hline Brain & 1 & 13 \\
\hline
\end{tabular}

distant sites for $5 \%$ of patients. Despite the changes in treatment approach, the relative distribution of recurrence sites is almost identical to that of our prior series (Table 2). ${ }^{10}$ In the current series, $72 \%$ of recurrences were local compared with $67 \%$ for our prior series; $53 \%$ were in the abdomen compared with $50 \%$ previously; $38 \%$ were in the CHT compared with $33 \%$ previously; and $7 \%$ were in other distant sites compared with $8 \%$ previously.

The observed relationship of recurrence location to stage generates hypotheses relevant to therapeutic strategy. IHT

TABLE 4. Multivariate analysis of time to local recurrence and local recurrence-free survival

\begin{tabular}{lcc}
\hline & $\begin{array}{c}\text { Time to local } \\
\text { recurrence } \\
\text { HR }(\mathbf{9 5} \% \mathbf{C I})\end{array}$ & $\begin{array}{c}\text { Local recurrence-free } \\
\text { survival } \\
\text { HR }(\mathbf{9 5} \% \mathbf{C I})\end{array}$ \\
\hline Non-epithelioid histology & $2.2(1.4-3.5)$ & $1.6(1.2-2.3)$ \\
HIOC & $0.7(0.4-1.1)$ & $0.7(0.5-1.0)$ \\
CTX & $0.7(0.5-1.1)$ & $0.7(0.5-1.0)$ \\
RT & $0.3(0.2-0.5)$ & $0.4(0.3-0.6)$ \\
\hline
\end{tabular}

$H R$, Hazard ratio; $C I$, confidence interval; $H I O C$, heated intraoperative chemotherapy; $C T X$, chemotherapy; $R T$, radiation therapy. remains the most common recurrence site independent of stage, likely representing regrowth of microscopic tumor not completely eradicated by treatment. Mediastinal, abdominal, and distant recurrence were observed more commonly with increasing stage, consistent with potential tumor erosion through diaphragm or pericardium or micrometastases disseminated via vascular or lymphatic routes that would presumably be more common with later stage tumors. The stage independence of apparent recurrence in the CHT suggests the possibility that some cases (particularly early stage) may represent new primary pleural disease.

As shown in Table 5, reports in the literature for local recurrence rates after EPP and RT (with or without neoadjuvant or adjuvant chemotherapy) range from $9 \%$ to $41 \% .^{3-6,10,18-26}$ One series reported a 2-year actuarial local recurrence rate of $53 \% .{ }^{24}$ It is difficult to compare recurrence rates across series for many reasons. The case mixes no doubt vary significantly in terms of other relevant factors, such as selection of patients for combined modality treatment, stage of disease, mesothelioma histology, margin status, reported results for intent to treat with multiple modalities versus for patients who actually received all treatments, length of follow-up, frequency of surveillance imaging, surgical expertise, chemotherapy agents, and RT technique. In addition, most of the reported rates are crude rates rather than actuarial rates, further rendering comparison difficult. Nonetheless, the majority of publications demonstrate high local recurrence rates.

The crude local recurrence rate for patients treated with EPP and RT in the current series was 39\%, which is among the highest reported. There are several potential explanations for this. A dedicated thoracic radiologist re-reviewed all post-treatment imaging to score recurrences. It is likely that many recurrences were asymptomatic, detected by imaging alone, and would not have been appreciated in earlier cohorts before the widespread use of CT and PET/CT for surveillance. In addition, the follow-up period in the current report is more than twice as long as most other series, allowing more time for recurrences to be detected. Last, there has been a learning curve in the evolution of adjuvant hemithoracic RT techniques. In the 2-dimensional RT planning era (before the availability of 3-dimensional CT planning), the approach at our institution was to deliver moderate dose RT, which consisted of $30 \mathrm{~Gy}$ to the hemithorax, $40 \mathrm{~Gy}$ to the mediastinum, and a boost dose to focal areas of concern to $54 \mathrm{~Gy}$. The crude local failure rate with this approach was $50 \%$, which is not surprising given that 30 Gy is likely an insufficient dose to achieve local control. ${ }^{18}$ In 2003, we started using high-dose EPT as developed at Memorial Sloan-Kettering Cancer Center. ${ }^{16}$ EPT delivers a higher dose of 54 Gy to the hemithorax and 39.6 Gy to the mediastinum; however, the technique results in some under-dosing at matched areas. In 2007, we reported a local recurrence rate with this approach of $27 \%$, and an updated 
TABLE 5. Patterns of failure after extrapleural pneumonectomy and radiation therapy (with or without neoadjuvant or adjuvant chemotherapy) for malignant pleural mesothelioma from several institutions

\begin{tabular}{|c|c|c|c|c|c|c|c|c|}
\hline & $\begin{array}{l}\text { No. treated } \\
\text { with EPP }\end{array}$ & $\begin{array}{c}\text { No. treated } \\
\text { with EPP } \\
\text { and RT } \\
\end{array}$ & Treatment & RT technique & $\begin{array}{l}\% \text { with } \\
\text { recurrence }\end{array}$ & LR \% & $\begin{array}{c}\text { Isolated } \\
\text { LR \% } \\
\end{array}$ & DR $\%$ \\
\hline Current series & 169 & $71 *(42 \%)$ & EPP, HIOC, CTX, RT & $\begin{array}{l}44 \% \text { EPT, } 30 \% \text { IMRT, } \\
11 \% \text { other }\end{array}$ & 75 & 39 & 15 & 53 \\
\hline Baldini and colleagues $^{10}$ & $46^{*}$ & $35(76 \%)$ & EPP, CTX, RT & 2D-RT & 54 & 35 & 13 & 41 \\
\hline Gupta and colleagues $^{19}$ & 86 & $78 *(91 \%)$ & EPP, RT & $\mathrm{EPT}$ & 74 & 41 & 19 & 55 \\
\hline Buduhan and colleagues $^{22}$ & 46 & $38 *(83 \%)$ & CTX, EPP, RT & $63 \%$ EBRT, $37 \%$ IMRT & 63 & 32 & 18 & 45 \\
\hline Gomez and colleagues $^{6}$ & 136 & $86 *(64 \%)$ & EPP, RT & IMRT & 62 & 16 & 2 & 59 \\
\hline de Perrot and colleagues ${ }^{3}$ & 45 & $30 *(67 \%)$ & CTX, EPP, RT & $\begin{array}{l}\text { IMRT } \\
\text { 3D-CRT }\end{array}$ & 53 & 17 & 17 & 37 \\
\hline Tonoli and colleagues ${ }^{25}$ & 56 & $56 *(100 \%)$ & EPP, RT, CTX & $\begin{array}{l}89 \% \text { IMRT } \\
7 \% \text { 3D-CRT } \\
4 \% \text { HT }\end{array}$ & 30 & 9 & 9 & 23 \\
\hline Krug and colleagues ${ }^{4}$ & $57 *$ & $40(70 \%)$ & CTX, EPP, RT & $\begin{array}{l}\text { EPT } \\
\text { IMRT }\end{array}$ & 40 & 19 & 14 & 26 \\
\hline Rea and colleagues ${ }^{20}$ & $17 *$ & $15(88 \%)$ & CTX, EPP, RT & EPT & 77 & 35 & NS & 65 \\
\hline Patel and colleagues ${ }^{24}$ & $30^{*}$ & $30 *(100 \%)$ & EPP, RT, CTX & IMRT & 73 & 33 & 13 & 60 \\
\hline van Sandick and colleagues ${ }^{23}$ & $15^{*}$ & $15 *(100 \%)$ & EPP, RT & IMRT & 73 & 33 & 9 & 67 \\
\hline Van Schil and colleagues $^{5}$ & 42 & $37 *(88 \%)$ & CTX, EPP, RT & 3D-CRT & NS & 16 & NS & 27 \\
\hline
\end{tabular}

$E P P$, Extrapleural pneumonectomy; $R T$, radiation therapy; $L R$, local recurrence; $D R$, distant recurrence (including abdomen and CHT); $H I O C$, heated intraoperative chemotherapy; $C T X$, chemotherapy; $I M R T$, intensity-modulated radiation therapy; $2 D-R T$, 2-dimensional radiation therapy; $E P T$, electron-photon technique; $E B R T$, external beam radiation therapy; $3 D$-CRT, 3-dimensional conformal radiation therapy; $H T$, helical tomotherapy; $N S$, not stated. *Patient cohort for reported recurrence results.

review of 34 patients treated with EPT has shown a local recurrence rate of $41 \%$ with the majority of recurrences occurring under blocks or at match lines in under-dosed areas. ${ }^{18,27}$ Investigators from Memorial Sloan-Kettering Cancer Center have reported local failure rates with EPT of $18 \%$ to $37 \%{ }^{16,26}$ Furthermore, the RT community has learned the importance of adequately extending the inferior border of the hemithoracic field to avoid "marginal misses" related to unappreciated extension of the pleura into the upper abdomen. ${ }^{16,18}$ In 2003, investigators from MD Anderson Cancer Center were the first to describe an IMRT technique that enables delivery of a high dose (50 Gy) to the entirety of the complex IHT volume. ${ }^{17}$ This IMRT technique appears to provide superior target coverage compared with prior techniques and should be associated with improved hemithoracic control rates. ${ }^{17,28}$ Only $13 \%$ of the current cohort received IMRT, which may be another explanation for the high local recurrence rate in this series. The early experience of IMRT at our institution resulted in unanticipated and unacceptable pulmonary toxicity, and for that reason, post-EPP IMRT treatment was suspended. ${ }^{29}$ More recently, other groups have demonstrated improved safety profiles with IMRT, and this approach should be reconsidered in future treatment strategies. ${ }^{6,24,30}$

\section{Study Limitations}

There are several limitations of this retrospective report. Although the intention to treat included evaluation for adjuvant chemotherapy and RT, the actual delivery of these modalities was highly variable. This was not due to a random or individualized treatment approach, but is a reflection of reality and the difficulties of safely delivering adjuvant therapies after aggressive surgery. Furthermore, because only the more fit patients typically were able to receive adjunctive treatments, it was not possible to assess the relative impact on the patterns of failure of adjuvant RT or chemotherapy compared with EPP alone. The analyses of local failure end points for patients treated with and without RT were provided for interest and to generate hypotheses for future trials. We acknowledge that formal conclusions cannot be drawn from these comparisons. Furthermore, not all of the recurrences were histologically confirmed. Chest x-ray was the prior standard follow-up modality for our earlier report, whereas the current series used CT or PET scans and specialized radiology review. As a result, many asymptomatic areas were detected and scored as recurrent disease, potentially inflating the recurrence numbers compared with other reports.

\section{CONCLUSIONS}

It is disappointing that our updated results failed to show a change in the patterns of failure after multimodality therapy with HIOC, pemetrexed-based chemotherapy, and more sophisticated RT, but others have similarly reported disappointing outcomes. MPM remains an aggressive disease that poses many treatment challenges. Careful study of large cohorts of patients managed with a standard 
intent to treat holds the only hope for progress. For patients with good performance status and localized disease, we believe that prolonged disease-free survival can be achieved only with aggressive local measures. We continue to support the role of surgery (EPP or pleurectomy/decortication as indicated to achieve MCR) for these patients. ${ }^{9,31}$ The optimal combination of therapies as adjuncts to surgery remains under active investigation. Given that the predominant pattern of failure remains local, implementation of postoperative hemithoracic RT is worthy of further study. However, the RT target volume is complex, in proximity to several radiosensitive critical organs, and early experiences with IMRT were associated with high fatality rates. ${ }^{21,29,32,33}$ More recent reports have demonstrated acceptable toxicity profiles for IMRT after EPP, but this approach is best used at experienced highvolume centers, and preferably on treatment protocols. $^{6,24,30}$ Alternatively, preoperative hemithoracic RT using hypo-fractionated IMRT is another option. Preliminary results for this technique are promising with respect to both efficacy and tolerability. ${ }^{34}$ Reports for intraoperative adjuncts such as HIOC and photodynamic therapy have shown suggestions of improved disease control in certain patient subpopulations, and these modalities are worthy of further investigation. ${ }^{13,35}$ HIOC is safe and feasible after $\mathrm{EPP}$, and with the discovery of more effective agents, local delivery in the context of minimal residual disease may represent an effective paradigm to enhance local control. Last, integration of neoadjuvant or adjuvant intravenous chemotherapy or new targeted agents is also warranted. Because there may be interactions between these various adjunctive treatment modalities, aggressive multimodality regimens are best tested on thoughtfully designed, institutional review board-approved protocols, and patients should be enrolled in prospective multicenter trials when feasible.

\section{Conflict of Interest Statement}

David E. Kozono reports consulting fees from Genzyme/ Sanofi. Raymond H. Mak reports consulting fees for Boehringer-Ingelheim, Inc and equity ownership in Celgene. Raphael Bueno reports consulting fees for Myriad Genetics, Inc, Novartis Inst. Biomedical Research, Verastem, Inc Study 1, Verastem, Inc Study 2, Castle Biosciences, Meso Study, Castle Biosciences, Esoph Study Genentech Inc, Siemens Medical Solutions, Inc and Exosome Diagnostics, Inc. All other authors have nothing to disclose with regard to commercial support.

\footnotetext{
References

1. Merritt N, Blewett CJ, Miller JD, Bennett WF, Young JE, Urschel JD. Survival after conservative (palliative) management of pleural malignant mesothelioma. J Surg Oncol. 2001;78:171-4

2. Vogelzang NJ, Rusthoven JJ, Symanowski J, Denham C, Kaukel E, Ruffie P, et al. Phase III study of pemetrexed in combination with cisplatin versus cisplatin
}

alone in patients with malignant pleural mesothelioma. J Clin Oncol. 2003;21: 2636-44.

3. de Perrot M, Feld R, Cho BC, Bezjak A, Anraku M, Burkes R, et al. Trimodality therapy with induction chemotherapy followed by extrapleural pneumonectomy and adjuvant high-dose hemithoracic radiation for malignant pleural mesothelioma. J Clin Oncol. 2009;27:1413-8.

4. Krug LM, Pass HI, Rusch VW, Kindler HL, Sugarbaker DJ, Rosenzweig KE, et al. Multicenter phase II trial of neoadjuvant pemetrexed plus cisplatin followed by extrapleural pneumonectomy and radiation for malignant pleural mesothelioma. J Clin Oncol. 2009;27:3007-13.

5. Van Schil PE, Baas P, Gaafar R, Maat AP, Van de Pol M, Hasan B, et al. Trimodality therapy for malignant pleural mesothelioma: results from an EORTC phase II multicentre trial. Eur Respir J. 2010;36:1362-9.

6. Gomez DR, Hong DS, Allen PK, Welsh JS, Mehran RJ, Tsao AS, et al. Patterns of failure, toxicity, and survival after extrapleural pneumonectomy and hemithoracic intensity-modulated radiation therapy for malignant pleural mesothelioma. J Thorac Oncol. 2013;8:238-45.

7. Treasure T, Lang-Lazdunski L, Waller D, Bliss JM, Tan C, Entwisle J, et al. Extra-pleural pneumonectomy versus no extra-pleural pneumonectomy for patients with malignant pleural mesothelioma: clinical outcomes of the Mesothelioma and Radical Surgery (MARS) randomised feasibility study. Lancet Oncol. 2011;12:763-72.

8. Weder W, Stahel RA, Baas P, Dafni U, de Perrot M, McCaughan BC, et al. The MARS feasibility trial: conclusions not supported by data. Lancet Oncol. 2011; 12:1093-4. author reply 1094-5.

9. Rusch V, Baldini EH, Bueno R, De Perrot M, Flores R, Hasegawa S, et al. The role of surgical cytoreduction in the treatment of malignant pleural mesothelioma: meeting summary of the International Mesothelioma Interest Group Congress, September 11-14, 2012, Boston, Mass. J Thorac Cardiovasc Surg. 2013;145:909-10.

10. Baldini EH, Recht A, Strauss GM, DeCamp MM Jr, Swanson SJ, Liptay MJ, et al. Patterns of failure after trimodality therapy for malignant pleural mesothelioma. Ann Thorac Surg. 1997;63:334-8.

11. Richards WG, Zellos L, Bueno R, Jaklitsch MT, Janne PA, Chirieac LR, et al. Phase I to II study of pleurectomy/decortication and intraoperative intracavitary hyperthermic cisplatin lavage for mesothelioma. J Clin Oncol. 2006;24:1561-7.

12. Tilleman TR, Richards WG, Zellos L, Johnson BE, Jaklitsch MT, Mueller J, et al. Extrapleural pneumonectomy followed by intracavitary intraoperative hyperthermic cisplatin with pharmacologic cytoprotection for treatment of malignant pleural mesothelioma: a phase II prospective study. J Thorac Cardiovasc Surg. 2009; 138:405-11.

13. Sugarbaker DJ, Gill RR, Yeap BY, Wolf AS, DaSilva MC, Baldini EH, et al. Hyperthermic intraoperative pleural cisplatin chemotherapy extends interval to recurrence and survival among low-risk patients with malignant pleural mesothelioma undergoing surgical macroscopic complete resection. J Thorac Cardiovasc Surg. 2013;145:955-63.

14. Sugarbaker DJ, Richards W, Bueno R. Extrapleural pneumonectomy in the treatment of epithelioid malignant pleural mesothelioma: novel prognostic implications of combined $\mathrm{n} 1$ and $\mathrm{n} 2$ nodal involvement based on experience in 529 patients. Ann Surg. 2014;260:577-82.

15. Sugarbaker DJ, Mentzer SJ, Strauss G. Extrapleural pneumonectomy in the treatment of malignant pleural mesothelioma. Ann Thorac Surg. 1992;54: 941-6.

16. Yajnik S, Rosenzweig KE, Mychalczak B, Krug L, Flores R, Hong L, et al. Hemithoracic radiation after extrapleural pneumonectomy for malignant pleural mesothelioma. Int J Radiat Oncol Biol Phys. 2003;56:1319-26.

17. Ahamad A, Stevens CW, Smythe WR, Vaporciyan AA, Komaki R, Kelly JF, et al. Intensity-modulated radiation therapy: a novel approach to the management of malignant pleural mesothelioma. Int J Radiat Oncol Biol Phys. 2003;55:768-75.

18. Allen AM, Den R, Wong JS, Zurakowski D, Soto R, Janne PA, et al. Influence of radiotherapy technique and dose on patterns of failure for mesothelioma patients after extrapleural pneumonectomy. Int J Radiat Oncol Biol Phys. 2007;68: 1366-74.

19. Gupta V, Krug LM, Laser B, Hudka K, Flores R, Rusch VW, et al. Patterns of local and nodal failure in malignant pleural mesothelioma after extrapleural pneumonectomy and photon-electron radiotherapy. J Thorac Oncol. 2009;4: 746-50.

20. Rea F, Marulli G, Bortolotti L, Breda C, Favaretto AG, Loreggian L, et al. Induction chemotherapy, extrapleural pneumonectomy (EPP) and adjuvant hemi-thoracic radiation in malignant pleural mesothelioma (MPM): Feasibility and results. Lung Cancer. 2007;57:89-95. 
21. Miles EF, Larrier NA, Kelsey CR, Hubbs JL, Ma J, Yoo S, et al. Intensitymodulated radiotherapy for resected mesothelioma: the Duke experience. Int $J$ Radiat Oncol Biol Phys. 2008;71:1143-50.

22. Buduhan G, Menon S, Aye R, Louie B, Mehta V, Vallieres E. Trimodality therapy for malignant pleural mesothelioma. Ann Thorac Surg. 2009;88:870-6.

23. van Sandick JW, Kappers I, Baas P, Haas RL, Klomp HM. Surgical treatment in the management of malignant pleural mesothelioma: a single institution's experience. Ann Surg Oncol. 2008;15:1757-64.

24. Patel PR, Yoo S, Broadwater G, Marks LB, Miles EF, D'Amico TA, et al. Effect of increasing experience on dosimetric and clinical outcomes in the management of malignant pleural mesothelioma with intensity-modulated radiation therapy. Int J Radiat Oncol Biol Phys. 2012;83:362-8.

25. Tonoli S, Vitali P, Scotti V, Bertoni F, Spiazzi L, Ghedi B, et al. Adjuvant radiotherapy after extrapleural pneumonectomy for mesothelioma. Prospective analysis of a multi-institutional series. Radiother Oncol. 2011;101:311-5.

26. Rusch VW, Rosenzweig K, Venkatraman E, Leon L, Raben A, Harrison L, et al. A phase II trial of surgical resection and adjuvant high-dose hemithoracic radiation for malignant pleural mesothelioma. J Thorac Cardiovasc Surg. 2001;122:788-95.

27. Mak K, Mannarino E, Richards WG, Mak RH, Chen AB, Kozono DE, et al. Patterns of local recurrence for mesothelioma patients treated with extrapleural pneumonectomy and radiation therapy using the electron-photon technique: the dosimetric challenges of electron-photon match lines and blocks. Int J Radiat Oncol Biol Phys. 2014;70:S608-9.

28. Krayenbuehl J, Oertel S, Davis JB, Ciernik IF. Combined photon and electron three-dimensional conformal versus intensity-modulated radiotherapy with inte- grated boost for adjuvant treatment of malignant pleural mesothelioma after pleuropneumonectomy. Int J Radiat Oncol Biol Phys. 2007;69:1593-9.

29. Allen AM, Czerminska M, Janne PA, Sugarbaker DJ, Bueno R, Harris JR, et al Fatal pneumonitis associated with intensity-modulated radiation therapy for mesothelioma. Int J Radiat Oncol Biol Phys. 2006;65:640-5.

30. Giraud P, Sylvestre A, Zefkili S, Lisbona A, Bonnette P, Le Pimpec Barthes F, et al. Helical tomotherapy for resected malignant pleural mesothelioma: dosimetric evaluation and toxicity. Radiother Oncol. 2011;101:303-6.

31. Sugarbaker DJ. Macroscopic complete resection: the goal of primary surgery in multimodality therapy for pleural mesothelioma. J Thorac Oncol. 2006;1: 175-6.

32. Rice DC, Smythe WR, Liao Z, Guerrero T, Chang JY, McAleer MF, et al Dose-dependent pulmonary toxicity after postoperative intensity-modulated radiotherapy for malignant pleural mesothelioma. Int J Radiat Oncol Biol Phys. 2007:69:350-7.

33. Kristensen CA, Nottrup TJ, Berthelsen AK, Kjaer-Kristoffersen F, Ravn J, Sorensen JB, et al. Pulmonary toxicity following IMRT after extrapleural pneumonectomy for malignant pleural mesothelioma. Radiother Oncol. 2009;92: 96-9.

34. Cho BC, Feld R, Leighl N, Opitz I, Anraku M, Tsao MS, et al. A feasibility study evaluating Surgery for Mesothelioma After Radiation Therapy: the "SMART" approach for resectable malignant pleural mesothelioma. J Thorac Oncol. 2014;9:397-402.

35. Friedberg JS, Mick R, Culligan M, Stevenson J, Fernandes A, Smith D, et al Photodynamic therapy and the evolution of a lung-sparing surgical treatment for mesothelioma. Ann Thorac Surg. 2011;91:1738-45.

Readers who found these articles interesting may also like to read the following papers found in recent and future issues of our sister publications, Seminars in Thoracic and Cardiovascular Surgery and Operative Techniques in Thoracic and Cardiovascular Surgery!

News and Views: Marc de Perrot. Novel induction therapies for pleural mesothelioma. Semin Thorac Cardiovasc Surg. Autumn 2014;26(3):192-200.

State of the Art: Yolonda Colson. Current Innovations in Sentinel Lymph Node Mapping for the Staging and Treatment of Resectable Lung Cancer. Semin Thorac Cardiovasc Surg. Autumn 2014;26(3):201-209.

State of the Art: Prasad Adusumilli. The IASLC/ATS/ERS Lung Adenocarcinoma Classification: What the surgeon should know. Semin Thorac Cardiovasc Surg. Autumn 2014;26(3):210-222.

Current Readings: Robert Suh. Percutaneous Ablation for Pulmonary Metastatic Disease. Semin Thorac Cardiovasc Surg. Autumn 2014;26(3):239-248.

Current Readings: Anne Tsao.Window-of-Opportunity Trials for Thoracic Malignancies. Semin Thorac Cardiovasc Surg. Expected publication April 2015.

State of the Art: Isabelle Schmitt-Opitz. Induction Therapy for Mesothelioma. Semin Thorac Cardiovasc Surg. Expected publication August 2015.

State of the Art: David Schrump. Precision Therapy for Lung Cancer: Tyrosine Kinase Inhibitors and Beyond. Semin Thorac Cardiovasc Surg. Expected publication August 2015. 